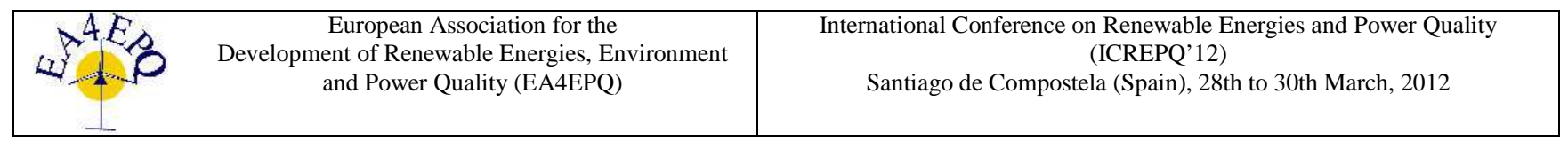

\title{
Power Quality Analyzer implemented with Matlab and Data Acquisition Toolbox
}

\author{
O. Pinzón-Ardila \\ Department of Electronic Engineering \\ Pontificia-Bolivariana University \\ Km 7 vía Piedecuesta, Bucaramanga (Colombia) \\ Phone:+0057 6796220 Ext 595, e-mail: omar.pinzon@upb.edu.co
}

\begin{abstract}
In this work was designed an equipment capable of monitoring electric grids and measure mayor perturbations that affect the grid. The prototype has it foundations on a Via EpiaMS embedded system, a data acquisition card, measurement probes (Voltage and Current), and software developed using MatLab's Data Acquisition Toolbox. The GUI provides a user friendly environment for visualization and management of the equipment. By other side the system allows to provide a briefing to the user where the times of the perturbations affecting the electrical grid are shown.
\end{abstract}

\section{Keywords}

Voltage gaps, Over-Voltages, flickers, Harmonics.

\section{Introduction}

In Colombia the Unidad de Planeación Minero Energético UPME (Unit of Mining and Energy planning) awaits within the long term to develop the Rational Use of Energy, in particular by emphasizing in the rigorous analysis of quality of power as an important issue that must be regulated by Comision Reguladora de Energía y Gas CREG (Regulatory Commission of Energy and Gas), to be compliant with the rational use of energy. By other side, several power quality measurement equipments enter Colombian market, but this kind of technology prevents further modifications or adaptation of measurement techniques, since they are proprietary type technologies. Thus, it is created a need to comprehend the concepts around these developments, to improve integration with existing equipment or to design new equipment capable to adapt to the needs of the industrial environment of the country. At current time, power quality measurement and analysis equipments are high-tech devices available at high costs. However, the reduction in costs mainly by hardware components makes it possible to study and implement of this kind of equipment in local research facilities.

\section{Design of Prototypes}

To design an electric power quality analyzer, it is necessary to take into account which are the most important perturbations, that need to be measured within the environment where the equipment is going to be used, because of that, regulatory policies must be taken into account, given that they provide design and measurement parameters to develop an electric power quality analyzer. In this work, for the construction of the measurement equipment, the following perturbations were chosen: undervoltage (Sags), transient overvoltages (Swells), flickers and Harmonic analysis on voltage and current signals.

By having defined clearly which the perturbations to be measured are, an analysis of the following regulations must be performed: (CREG 024-2005) and (CREG 0162007) imposed by the CREG (Regulatory Commission of Energy and Gas in Colombia) and its recommendations must be followed to develop equipment compliant with government requirements regarding electric perturbation measurements.

\section{A. Analysis and Design (Sags)}

Regulation CREG 024-2005 dictates the following: "sag, is a voltage fluctuation known by producing a voltage transient depression in respect to the standard wave, in a point of the sinus. It is considered a sag, that sag which last 0.5 cycles per 1 minute, with a magnitude equal or less than $90 \%$ of the RMS value of the wave (CREG 2005)"

The measurement algorithm is shown in Figure 1, where a signal it's acquired by means of a data acquisition system which will be explained later in this work. The voltage level must be selected where perturbations are going to be measured.

Report generation is shown In Figure 2, once the voltage comparison shown in Figure 1 is obtained, the process of measurement of the time since the sag starts until it ends is made, then a report is given to the user showing beginning and final times and voltage magnitude (IEC61000-2-2. (2002)). 


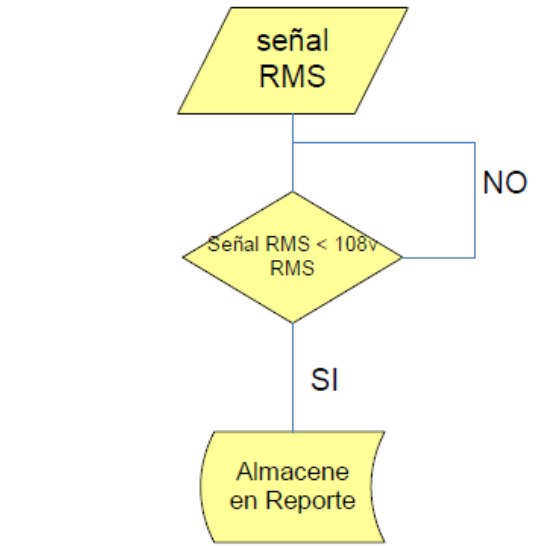

Fig.1. Sags Comparison Algorithm 120V RMS.

Report generation is shown In Figure 2, once the voltage comparison shown in Figure 1 is obtained, the process of measurement of the time since the sag starts until it ends is made, then a report is given to the user showing beginning and final times and voltage magnitude (IEC61000-2-2. (2002)).

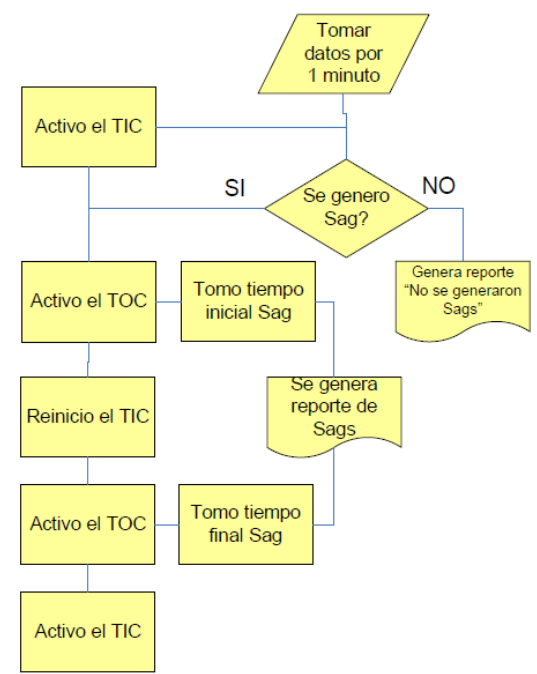

Fig. 2. Report Generation.

Figure 3 shows Graphic User Interface (GUI) made for selection of sag's parameters measurement.

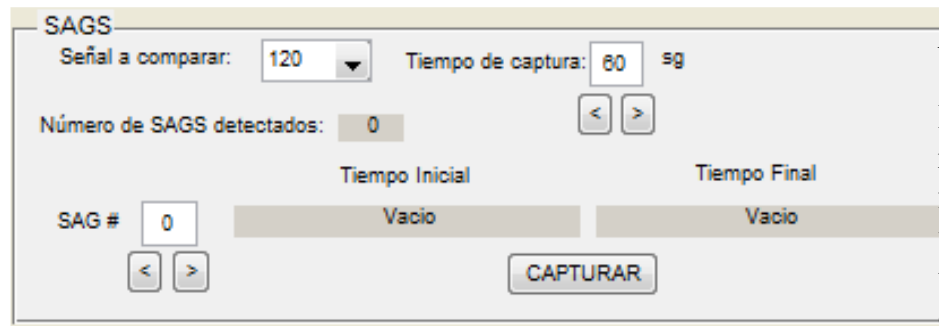

Fig 3. GUI Sag's measurement.

\section{Analysis and Design (Swell)}

Regulation (CREG 024-2005) recommendation for Swell is as follows: "A swell is a voltage fluctuation featured by a transient increment of voltage in respect to the standard wave, in a pulse of the sinus function. Voltage peaks are considered Swells when they occur within 0.5 cycles per 1 minute, with magnitude above $10 \%$ of the RMS value of the wave (CREG 2005)". Taking into account what the regulation suggests, then a comparison is made between the RMS signal acquired by the equipment and a theoretical value, then the developed algorithm is applied. Figure 4 shows the comparison algorithm developed for Swell analysis.

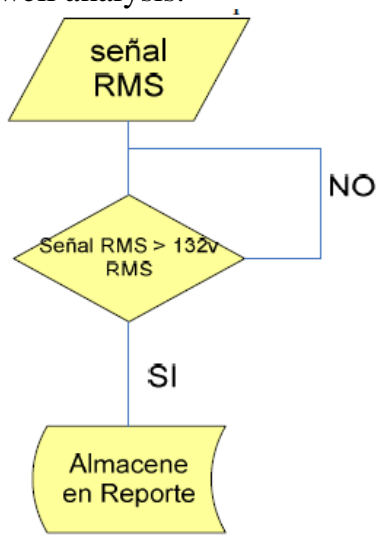

Fig. 4. Swell Comparison Algorithm 120V RMS.

Once the signal's comparison block value is obtained, an algorithm for initial time, final time and Swell perturbation magnitude measurement is applied; this algorithm follows the same methodology in a similar measurement of Sags (IEC61000-4-30 (2003)). See Figure 2.

Figure 5 shows swell's GUI, which allows variation of time parameters for network analysis, variation of several voltage levels in which measurements can be performed; and besides that, it can be made a report of the different Swell detections during the period of measurement.

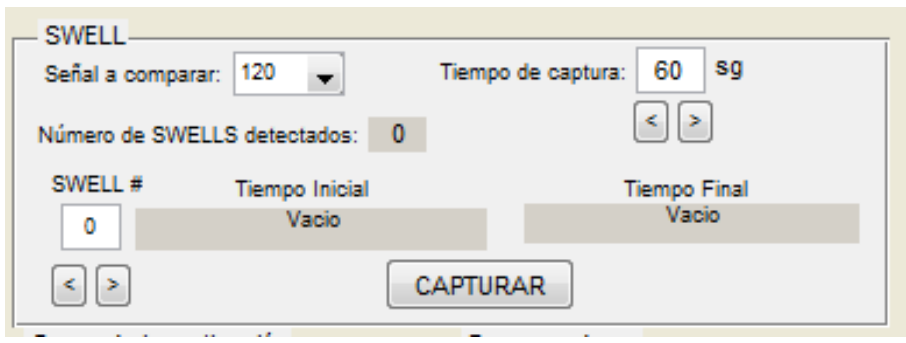

Fig 5. GUI Swells' mēasurement.

\section{Analysis and Design (Flickers)}

Regulation (CREG 024-2005), in respect to Flickers recommends: "Flicker is the impression of instability from visual sensation caused by luminescent stimulus, whose luminescence or spectral distribution fluctuates in time. The flicker is measured by a perceptibility indicator of an equipment or system under voltage fluctuations, during a short time period (about ten minutes), and it is statistically obtained from voltage's signal treatment. The definition on how to calculate it is in IEC-61000-4-15 standard; this short period of time is named PST (CREG (024-2005))".

Once voltage is obtained by the equipment, then the following algorithm is developed as shown in Figure 6. 


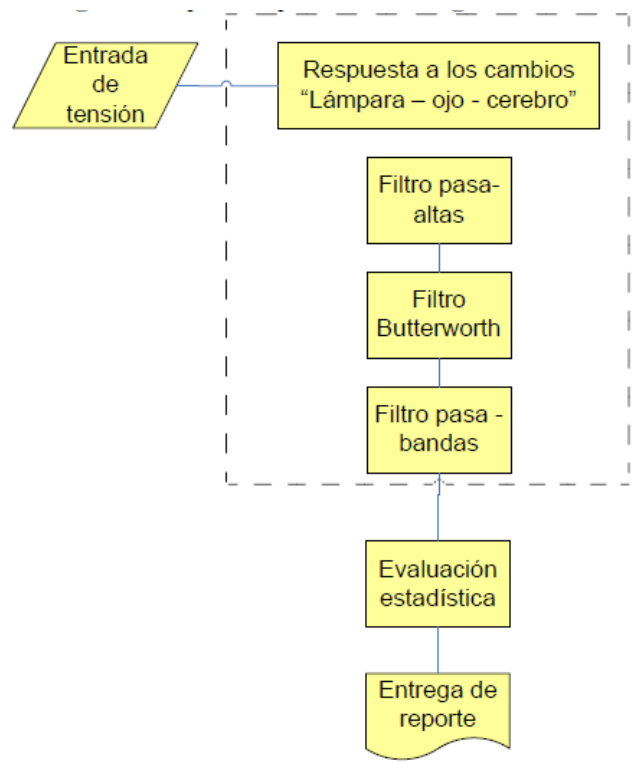

Fig. 6. Flicker measurement.

The first section of this algorithm consists in the use of a series of filters. First filter is a first order high-pass filter with a cut-off frequency at around $0.05 \mathrm{~Hz}$ with a $-3 \mathrm{~dB}$ attenuation, and it is intended to eliminate input signal's CC components (IEC61000-4-15 (2003)).

The second is a sixth order Butterworth filter, with a cutoff frequency of $42 \mathrm{~Hz}$ with a $-3 \mathrm{~dB}$ attenuation, and it eliminates the high frequency ripple at demodulator's output, this ripple merges by multiplying demodulator's input signal by itself (IEC61000-4-15 (2003)).

The third filter consists of a band-pass filter, which emulates human reactions towards luminescent variations flux, its cut-off frequency is $8.8 \mathrm{~Hz}$, and it is described by IEC 61000-4-15 standard with the following transfer function, shown in (1):

$$
f_{(s)}=\frac{K \omega_{1} s}{s^{2}+2 \lambda s+\omega_{1}^{2}} * \frac{1+\frac{s}{\omega_{2}}}{\left(1+\frac{s}{\omega_{3}}\right)\left(1+\frac{s}{\omega_{4}}\right)}
$$

Once these filters are applied to the signal, then a percentile statistical analysis is performed. Having stipulated the cumulated frequency values, the momentary impression level (Flicker), is then evaluated with (2).

$$
P_{s \neq}=\sqrt{0.0314 P_{01, \mathrm{~g} g}+0.0525 P_{10, \mathrm{~g} g}+0.0657 P_{30, \mathrm{~g} g}+0.28 P_{10, \mathrm{~g}}+0.08 P_{50, \mathrm{~g}}}(2)
$$

The evaluation method consists of a smoothing method where the following values are determined:

$$
\begin{aligned}
& P_{0.1 ; g}=P_{0.1} \\
& P_{1.0 ; g}=\left(\frac{1}{3}\right)\left(P_{0.7}+P_{1.0}+P_{1.5}\right) \\
& P_{3.0 ; g}=\left(\frac{1}{3}\right)\left(P_{2.2}+P_{3.0}+P_{4.0}\right) \\
& P_{10 ; g}=\left(\frac{1}{5}\right)\left(P_{6.0}+P_{8.0}+P_{10}+P_{13}+P_{17}\right) \\
& P_{50 ; g}=\left(\frac{1}{3}\right)\left(P_{30}+P_{50}+P_{60}\right)
\end{aligned}
$$

A variable of mayor relevance to evaluate the flicker phenomena is provided by Long Term Flicker Plt (Schlabbach - 2000); shown in (3).

$$
P_{l t}=\sqrt[3]{\left(\frac{1}{N}\right) \sum_{i=1}^{N} P_{s t i}^{3}}
$$

For this assessment, high level flicker are specially evaluated and observation period of time is usually 2 hours $(\mathrm{N}=12)$. The PLT is determined from a sliding measurement interval (Schlabbach - 2000). The advantage of this procedure is the direct transfer of voltage fluctuations in several forms and amplitudes in an evaluation number. Figure 7 shows flicker's analysis GUI.

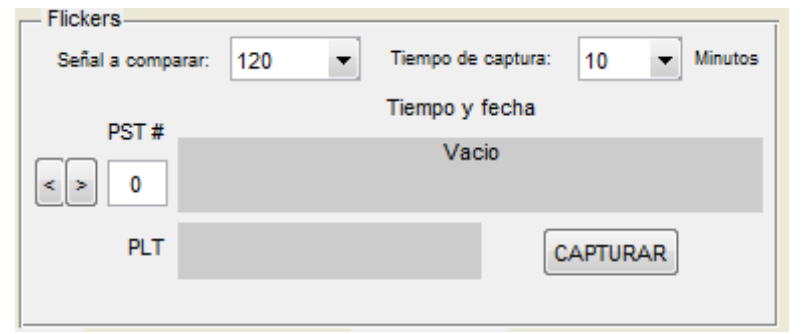

Fig. 7. Flickers analysis.

\section{E. Analysis and Design (Harmonics)}

Regulation (CREG 024 - 2005), recommends the following in regards of THDV: "It is an indicator of Total Harmonic Distortion of Voltage, in respect to the standard waveform expressed in a percentage. The procedure on how to calculate is defined in IEEE 519 [1992] standard (CREG (024-2005))". To obtain THDV it is necessary to calculate system's harmonic components. Figure 8 shows the algorithm's block diagram that must be followed to obtain THDV.

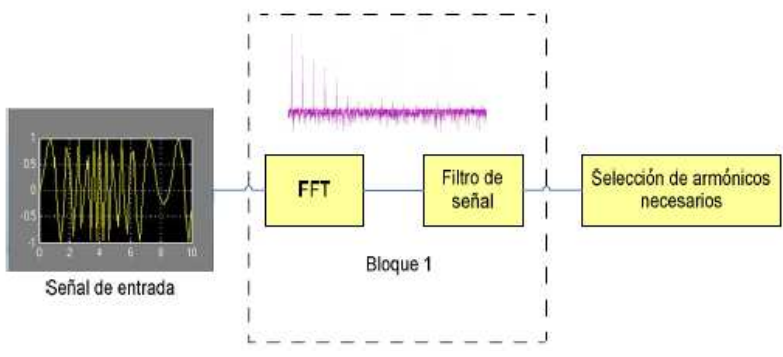

Fig. 8. Harmonic acquisition.

Once the signal has been acquired, it is processed with a Fast Fourier Transform (FFT), then a filter is applied just 
where harmonic components are obtained from the acquired signal, the final step is to select harmonic components that are multiples the fundamental frequency until the fortieth harmonic is obtained (Arrillaga - 1985). In this case inter-harmonic components are neglected in the analysis of harmonics. The algorithm used to calculate the distortion factor and THDV is shown in Figure 9.

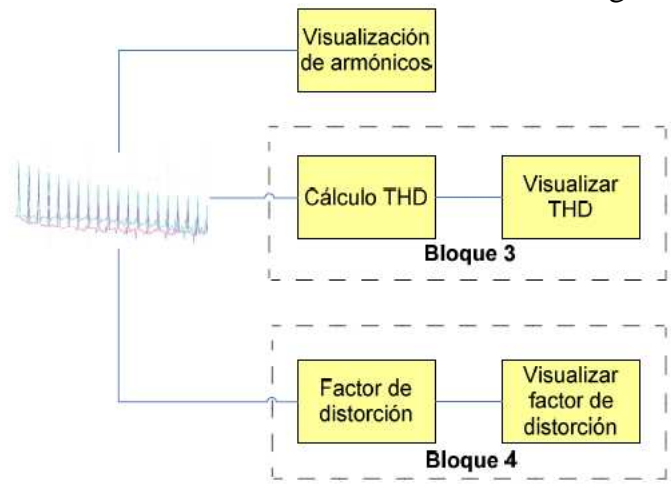

Fig 9. THDV analysis.

The value of total harmonic distortion is the relation between the rms value (effective value) of all corresponding terms to frequencies different from the fundamental and the rms value of the corresponding term to the fundamental frequency (Arrillaga - 1985).

The equation to obtain THDV is shown in (4):

$$
\% T H D V=\frac{\sqrt{I_{\text {arm }_{\text {mus }}}^{2}+I_{d c}^{2}}}{I_{\text {fundamental }_{\text {rms }}}} * 100
$$

The last step is to calculate the Distortion Factor (DF) that indicates the reduction of power factor in presence of harmonic within the system. (Arrillaga - 1985).

Distortion factor can be obtained using (5):

$$
F D=\sqrt{\frac{1}{1+(T H D V)^{2}}}
$$

Figure 10 shows the GUI designed for harmonic's analysis and visualization in the equipment.

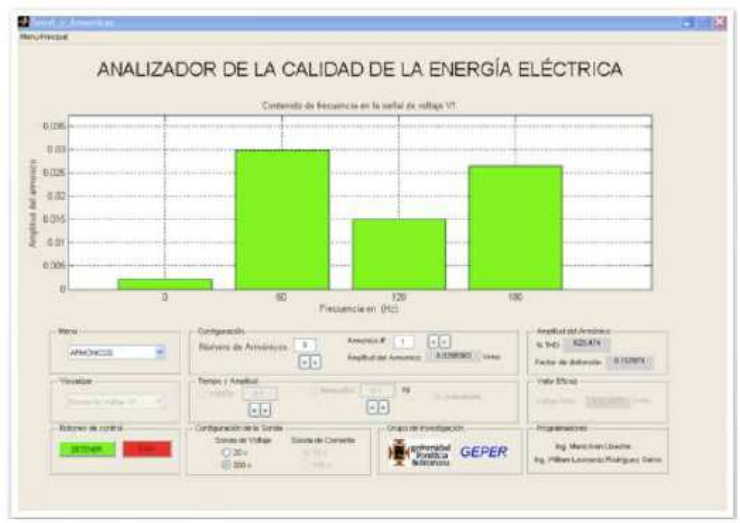

Fig. 10. GUI for Harmonics analysis.

\section{Description of the Experimental Plataform}

The final prototype has five modules which are conjugated in a single embedded measurement system. The first module is formed by current and voltage measurement probes. See figures 11 and 12 .

Voltage probes: FLUKE's DP120 probes are differential voltage probes that allow performing measurements of high voltages, some of its measurement features are: $600 \mathrm{~V}$ category III and $1000 \mathrm{~V}$ category II. Features of maximum dynamic differential voltage are: $1000 \mathrm{VCC}$, $1000 \mathrm{Vrms}, 1200 \mathrm{~V}$ (DC+AC peak).

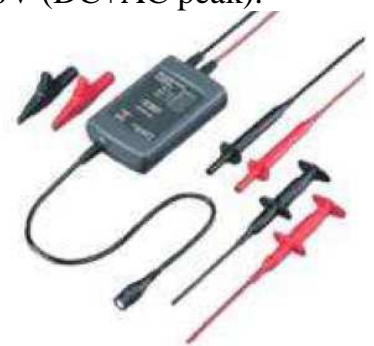

Fig. 11. Voltage probe FLUKE DP 120

Source: http://us.fluke.com/fluke/usen/Accessories/ est- LeadsProbes-anClips/DP120.htm?PID=55940

Current probes: FLUKE's 80i-110s probes are CA/CC current probes of amperimetric clamp type, it is designed to represent current waveforms in modern industrial and commercial electric energy distribution systems. Average current measurement ranges are: 0 to a maximum of $100 \mathrm{~A}$ in $\mathrm{DC}$ or $\mathrm{AC}$.

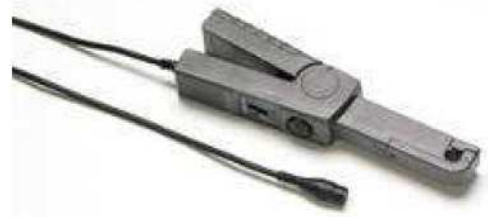

Fig. 12. Current probe: FLUKE 80i-110s

Source: http://us.fluke.com/fluke/usen/Accessories/Current Clamps/80i-110s.htm?PID=55352

Then, there is the signal filtering module. This is a six channel card where the input signal is put in to BNC connectors, and then it goes across a signal amplification stage by the use of AD8031 integrated circuit, this circuit offers a high speed performance and small signal bandwidth of $80 \mathrm{MHz}$. Its lo distortion factor and quick setting time makes it an ideal ADC buffer.

After passing through the amplification stage, the signal is then sent to a filtering stage, using the LTC1569-7, which is a tenth order low-pass filter with linear phase. Its high selectivity plus its linear phase within the passband make it appropriate for the filtering task in data communication and transferring as well as in data acquisition systems. See Figure 13.

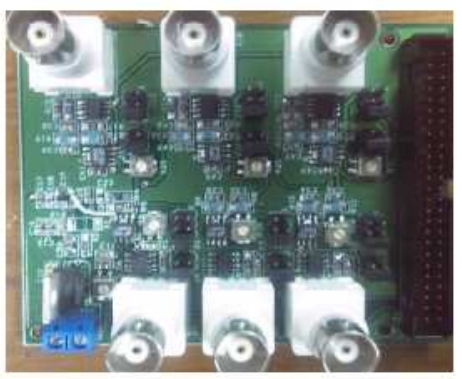

Fig. 13. Signals Conditioning Card. 
The data acquisition card DAS6036 Measurement Computing is found in the third module, this card has 16 A/D channels with 16 bit resolution, a maximum sampling speed of $200 \mathrm{KS} / \mathrm{s}$, two 16 bit A/D channels at $10 \mathrm{KS} / \mathrm{S}$ amongst other features. The decision to choose this card lies in MatLab's Data Acquisition Toolbox drivers which allow an outstanding communication between software and hardware. See Figure 14.

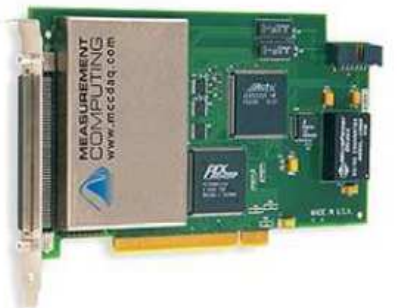

Fig. 14. Data Aquisition Card.

Source: http://www.mccdaq.com/pci-data acquisition/PCIDAS6036.aspx

There is, in the fourth module the processing card. A Via Epia MS card is used, this is a low cost card, but it has the performance that is necessary for the excellent functioning of the equipment. The Via Epia Ms card has one $1 \mathrm{GHz}$ processor with a extremely low energy consumption level and a silent operation. This card offers an improved digital performance through the VIA chipset with an integrated MPEG-2 graphics accelerator VIA CLE266 2D/3D UniChrome. See Figure 15.

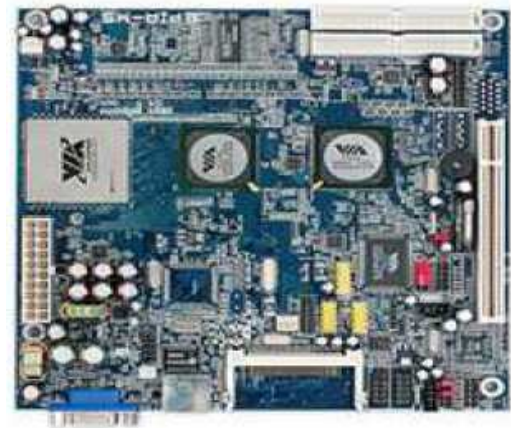

Fig. 15. VIA Epia MS Card.

Source: http://www.via.com.tw/en/products/mainboards/d wnloads.jsp?motherboard_id=281

The last module is the visualization screen of the equipment, a Mimo touch screen was selected for this prototype, and it provides an interactive and smooth visualization with the user. See Figure 16.

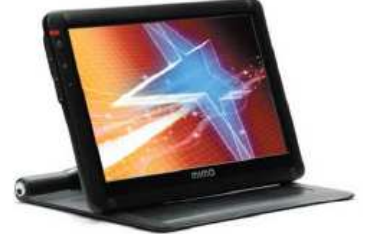

Fig. 16. Mimo Touch Screen

Source: http://www.mimomonitors.com/

Finally the equipment developed by EPER research group of Universidad Pontificia Bolivariana seccional Bucaramanga can be appreciated. See Figure 17.

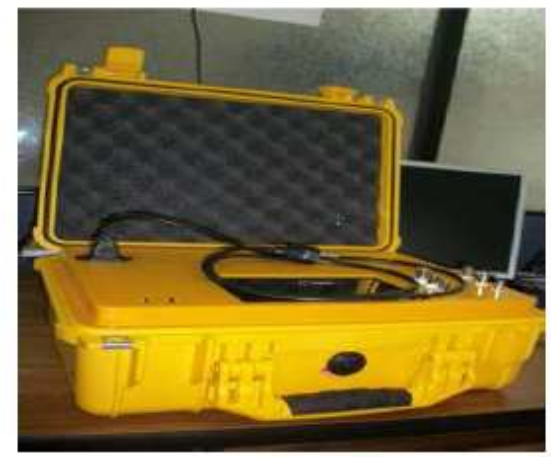

\section{Conclusion}

Fig. 17. Final Prototype.

In this work different electric grid perturbations were analyzed and the most important of them were measured by developing a measuring algorithm for each one of those perturbations such as Sags, Swells, Flickers, and Harmonic Distortions. All those algorithms were created regarding international and national regulations and aiming to develop a device with a high level and capability of measurement at a low cost.

A robust experimental platform was implemented, capable of executing all proposed algorithms, and compliant with electromagnetic compatibility and industrial security regulations, in order to obtain an equipment of development able to adapt to Colombian industry.

Within the development of the electric power quality measurement equipment, it remains open the possibility of new updates depending on the modifications to quality regulations and standards that could be made in the future.

\section{References}

[1]. Akagi, H, (1996) Trends in active power line conditioner. IEEE Transactions on Power Electronic.

[2]. Akagi, H., Watanabe, H. and Aredes, M. (2007). Instantaneous Power Theory and Applications to Power Conditioning. New York. IEEE Press.

[3]. Arrillaga. J.,Bradley, D. A., and Bodger. P. S. (1985). Power System Harmonics Analysis. Jhon Wiley and sons.

[4]. Arrillaga, J, Watson , N, R, and Chens , S. (2000). Power System Quality Assessment. Jhon Wiley and Sons.

[5]. Schlabbach. J, Blume. D, S. (2000). Voltage Quality in Electrical Power Systems. The Institution of Engineering and Technology.

[6]. EN-50160. (1996). Características de la Tensión Suministrada Por Las Redes Generales de Distribución, UNE.

[7]. IEC61000-1-1. (1992). Electromagnetic Compatibility (EMC) - Part 1: General - Section 1: Application and Interpretation of Fundamental Definitions and Terms, Technical Report IEC - 61000-1-1. IEC.

[8]. IEC61000-2-2. (2002). Electromagnetic Compatibility (EMC) - Part 2-2: Environment - Compatibility Levels for Low-Frequency Conducted Disturbances and Signalling in Public Low-Voltage Power Supply Systems, International Standard 61000-2-2. IEC.

[9]. IEC61000-4-30. (2003). International Standard IEC 61000-4-30, Electromagnetic Compatibility (EMC) - Part 
4-30: Testing and measurement techniques power quality measurement methods. IEC.

[10].IEEE Std 519-1992. (1992). Recommended Practices and Requirements for Harmonic Control in Electrical Power System. IEEE.

[11].IEC61000-4-15 (2003). Compatibilidad electromagnetic (CEM) -Parte 4 Técnicas de ensayo y medida. Sección 15. Medidor de Flicker.

[12].CREG (024-2005). Norma de calidad de potencia eléctrica aplicables a los servicios de Distribución de Energía Eléctrica. 